\title{
Acute Intestinal Distress Syndrome Following Laparoscopy
}

\author{
Darren J. Porter ${ }^{\mathrm{a}, \mathrm{b}}$, Jane Kilkenny ${ }^{\mathrm{a}}$, Sam Greensmith ${ }^{\mathrm{a}}$, \\ Pradeep Patila
}

\begin{abstract}
Intestinal ischemia is a rare but potentially fatal complication of laparoscopic surgery. Acute intestinal distress syndrome is the term used to describe intestinal ischemia that results from laparoscopic procedures in patients with pre-existing cardiovascular risk factors. We present a case of fatal intestinal ischemia due to acute intestinal distress syndrome. To avoid this potentially fatal complication of laparoscopic surgery, thorough pre-operative assessment should be performed to identify patients with cardiovascular and respiratory disease who are at increased risk of this condition. Senior surgical and anesthetic staff should be involved in laparoscopic procedures on these patients and prolonged procedures in the Trendelenburg position with highpressure pneumoperitoneum should be avoided or minimized.
\end{abstract}

Keywords: Mesenteric ischemia; High-pressure pneumoperitoneum; Trendelenburg position

\section{Introduction}

Intestinal ischemia is a rare but potentially fatal complication of laparoscopic surgery. Acute intestinal distress syndrome is a term that describes intestinal ischemia due to the physiological changes associated with laparoscopy. Acute intestinal distress syndrome is most likely to occur in patients with pre-existing cardiovascular and respiratory disease who undergo prolonged laparoscopic procedures in the Trendelenburg position with high-pressure $\mathrm{CO}_{2}$-induced pneumoperitoneum.

In this study, we describe a case of a patient with vascular risk factors who developed a fatal occlusion of the celiac trunk and superior mesenteric artery (SMA) following a combined laparoscopic cholecystectomy and bilateral salpingo-oophorectomy (BSO).

\section{Case Report}

A 54-year-old lady was transferred from another hospital to

Manuscript submitted July 24, 2017, accepted August 7, 2017

${ }^{a}$ Ninewells Hospital and Medical School, Dundee, Scotland, UK

${ }^{\mathrm{b}}$ Corresponding Author: Darren J. Porter, Ninewells Hospital and Medical School, Dundee, Scotland, UK. Email dporter@tcd.ie

doi: https://doi.org/10.14740/jmc2884w the vascular service following a $\mathrm{CT}$ of abdomen and pelvis that demonstrated acute mesenteric ischemia with signs of occlusion of the celiac axis and SMA with a patent inferior mesenteric artery. The CT also demonstrated intestinal edema with possible segmental short bowel wall necrosis with pneumatosis intestinalis at the proximal jejunum. No significant intra-peritoneal fluid and no signs of perforation were demonstrated at that time. This patient was day 7 after combined laparoscopic cholecystectomy and BSO.

She had initially been admitted with a 24-h history of colicky right upper quadrant (RUQ) pain on a background of a 6-month history of colicky post-prandial peri-umbilical pain. She had a background medical history of COPD, hypertension and hypercholesterolemia, but no history of ischemic heart disease, diabetes mellitus or peripheral vascular disease. Previous surgical history included an open appendicectomy and a hysterectomy for menorrhagia secondary to uterine fibroids. This patient was a heavy smoker, with an 80 pack year smoking history.

On the night of admission, she became unwell with increased RUQ pain, nausea and fevers. On examination, Murphy's sign was positive. She was tachycardic and pyrexic with elevated inflammatory markers. An urgent $\mathrm{CT}$ of abdomen and pelvis was arranged and this demonstrated acute cholecystitis. The patient was treated with intravenous antibiotics and was consented for a laparoscopic cholecystectomy.

Since she was awaiting an elective laparoscopic BSO, the decision was taken to perform this procedure at the same time as the laparoscopic cholecystectomy. Whilst there were no documented difficulties with either procedure, the duration of the combined laparoscopic procedure was $3 \mathrm{~h}$.

On day 2 postoperatively, the patient complained of further abdominal pain, and was noted to be tachycardic and hypotensive. Bloods were taken and demonstrated a neutrophil leucocytosis of $30.5 \times 10^{9} / \mathrm{L}(3.6-11.0)$, sodium of $130 \mathrm{mmol} / \mathrm{L}$ (133- $147 \mathrm{mmol} / \mathrm{L})$, an amylase of $262 \mathrm{IU} / \mathrm{L}(<100 \mathrm{IU} / \mathrm{L})$, and a CRP of $380 \mathrm{mg} / \mathrm{L}(<8 \mathrm{mg} / \mathrm{L})$, but normal renal function, liver function and lactate. A repeat CT scan was requested and demonstrated "a small bowel obstruction" with no evidence of free intra-abdominal gas or fluid, which was treated conservatively with nil orally, an NG tube and IV fluids.

Five days later, the patient complained of severe generalized abdominal pain. Given the severity of this patient's pain and high analgesic requirements, a CT of abdomen and pelvis was arranged and demonstrated an occluded SMA (Fig. 1) with evidence of ischemia of the distal ileum and ascending colon. The patient was transferred urgently to a tertiary referral center for joint general surgical and vascular surgery input. 


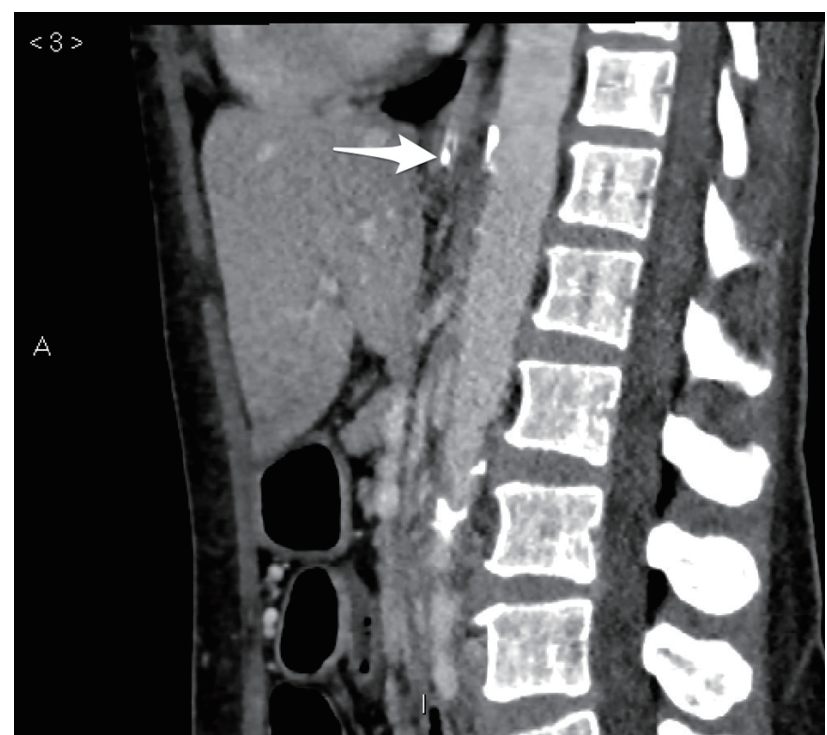

Figure 1. CT of abdomen and pelvis demonstrating acute mesenteric ischemia secondary to occlusion of the superior mesenteric artery (white arrow).

On arrival at the tertiary referral center, the patient was hemodynamically unstable; she was resuscitated and brought to theater urgently. A midline laparotomy was performed which demonstrated gangrenous distal ileum and ascending colon and the SMA was exposed. Proximal and distal control of the SMA was obtained and an angiogram was performed which confirmed SMA occlusion. The SMA was stented and the post-stent angiogram demonstrated good perfusion through the SMA. The distal $20 \mathrm{~cm}$ of ileum and the ascending colon were divided with a linear stapler and sent to histopathology. There were four areas of patchy necrosis from the mid jejunum to the proximal ileum, which were over-sewn. The patient was unstable throughout the procedure and hence neither an ileocolic anastomosis nor stoma formation was performed. The abdomen was closed with a scheduled relook laparotomy for $48 \mathrm{~h}$ postoperatively.

Postoperatively, the patient was transferred to the intensive care unit where she required minimal inotropic support.

During the relook laparotomy, the remaining small intestine with the areas that were over-sewn appeared healthy on inspection and an ileostomy was formed. The patient thereafter made a slow but steady postoperative recovery and was transferred from intensive care to the surgical high dependency unit 5 days later.

Unfortunately 1 week later the patient complained of severe abdominal pain. Inflammatory markers were elevated and an arterial blood gas revealed a metabolic acidosis with a $\mathrm{pH}$ of $7.1(7.35-7.45)$ and a lactate of $14.8 \mathrm{mmol} / \mathrm{L}(<2.0 \mathrm{mmol} / \mathrm{L})$. A laparotomy was performed and $1,600 \mathrm{~mL}$ of small bowel content was found within the peritoneal cavity. Multiple areas of small bowel necrosis were found with two small bowel perforations. An extensive small bowel resection was undertaken with only $30 \mathrm{~cm}$ of viable proximal small bowel left. Postoperatively, the patient was transferred to ICU, with high inotropic requirements failing to maintain her blood pressure, and she unfortunately died $24 \mathrm{~h}$ later.

\section{Discussion}

Laparoscopic surgery aims to achieve a satisfactory therapeutic result whilst minimizing the traumatic and metabolic stress of the intervention. Tissue trauma is significantly less than that associated with open surgical procedures resulting in many advantages, such as reduced postoperative pain, smaller wounds, and lower rates of wound complications and these are associated with shorter hospital stay, more rapid return to normal activity and reduced cost of health care [1].

Despite these clear advantages, laparoscopic surgery may result in serious complications due to the important physiological changes that occur during the procedure. In complicated surgical cases of extended duration, such as in the case presented, the prolonged Trendelenburg position and carbon dioxide insufflation result in a pneumoperitoneum that often has adverse hemodynamic and respiratory consequences [2].

The Trendelenburg position is commonly used for gynecological procedures, such as a laparoscopic BSO. In a study by Russo et al, the Trendelenburg position was found to increase central venous pressure, pulmonary capillary wedge pressure and pulmonary arterial pressures and decrease cardiac output. Pneumoperitoneum increases these pressures further and thus the combination of a prolonged procedure, the Trendelenburg position and high $\mathrm{CO}_{2}$ pneumoperitoneum reduces cardiac output, blood pressure and hence causes reduced tissue perfusion [2]. The Trendelenburg position and high-pressure pneumoperitoneum can also increase intra-abdominal pressure, which may also reduce mesenteric perfusion and cause ischemia [3].

Normal intra-abdominal pressure is $5-7 \mathrm{~mm} \mathrm{Hg}$. At an intra-abdominal pressure of $12 \mathrm{~mm} \mathrm{Hg}$ renal impairment develops [4]. The intra-abdominal perfusion pressure is the result of mean arterial blood pressure minus intra-abdominal pressure [4]. Hence a reduced mean arterial blood pressure due to the Trendelenburg position and the pneumoperitoneum and an elevated intra-abdominal pressure due to pneumoperitoneum can predispose to splanchnic ischemia during laparoscopic surgery; this is especially the case in prolonged procedures [5]. To further compromise the splanchnic circulation carbon dioxide (used to create the pneumoperitoneum) is absorbed into the circulation, resulting in hypercapnia, respiratory acidosis and this increases the systemic vascular resistance secondary to the hemodynamic stress response [6]. This splanchnic hypoperfusion due to the physiological changes associated with laparoscopy has been termed "acute intestinal distress syndrome" [7]. Acute intestinal distress syndrome may be further exacerbated by pre-exiting mesenteric ischemia as was likely the case in the patient described [8].

Although these physiological changes can theoretically compromise splanchnic perfusion, they are well tolerated in healthy patients with normal cardiovascular and pulmonary function with no clinical sequelae. Risk factors such as atherosclerosis can predispose to mesenteric ischemia [9]. In the case described the patient was a heavy smoker, hypertensive and hypercholesterolemic and it is highly likely, although un- 
proven, that she had pre-existing mesenteric atherosclerotic disease, and this would have predisposed her to acute intestinal distress syndrome secondary to prolonged laparoscopic surgery.

It is also plausible that the 6-month history of colicky post-prandial pain was caused by chronic mesenteric ischemia and not cholelithiasis.

Although acute intestinal ischemia is regarded as a rare complication of laparoscopic surgery, it is associated with a $71 \%$ mortality in reported cases [10]. For this reason, careful patient selection for laparoscopic surgery should be undertaken, the surgeon should be competent and efficient avoiding prolonged procedures especially with the patient in the Trendelenburg position and high-pressure pneumoperitoneum should be avoided especially in patients who have documented or suspected mesenteric vascular disease.

Gasless laparoscopy using abdominal wall elevating devices has been considered in an attempt to avoid the adverse physiological effects of $\mathrm{CO}_{2}$ pneumoperitoneum that can occur in standard laparoscopy. This technique might be considered in high-risk patients with cardiovascular or respiratory disease [11].

\section{Conclusion}

Acute intestinal distress syndrome is a rare complication of laparoscopic surgery. It tends to occur in high-risk patients with significant cardiovascular and respiratory comorbidity who undergo prolonged laparoscopic procedures with high intra-abdominal pressures in the Trendelenburg position.

Thorough pre-operative assessment should be undertaken to identify "at risk" patients and senior anesthetists and surgeons should be involved in laparoscopic procedures in these patients. Prolonged operative times especially in the Trendelenburg position with high-pressure pneumoperitoneum should be avoided or minimized to reduce the risk of this rare but potentially fatal complication.

\section{Grant}

None.

\section{Conflicts of Interest}

We have no conflicts of interest to declare.

\section{References}

1. Levy BF, Tilney HS, Dowson HM, Rockall TA. A systematic review of postoperative analgesia following laparoscopic colorectal surgery. Colorectal Dis. 2010;12(1):515.

2. Russo A, Marana E, Viviani D, Polidori L, Colicci S, Mettimano M, Proietti R, et al. Diastolic function: the influence of pneumoperitoneum and Trendelenburg positioning during laparoscopic hysterectomy. Eur J Anaesthesiol. 2009;26(11):923-927.

3. Diebel LN, Dulchavsky SA, Wilson RF. Effect of increased intra-abdominal pressure on mesenteric arterial and intestinal mucosal blood flow. J Trauma. 1992;33(1):45-48; discussion 48-49.

4. Cheatham ML, De Waele J, Kirkpatrick A, Sugrue M, Malbrain ML, Ivatury RR, Balogh Z, et al. Criteria for a diagnosis of abdominal compartment syndrome. Can J Surg. 2009;52(4):315-316.

5. Sammour T, Mittal A, Loveday BP, Kahokehr A, Phillips AR, Windsor JA, Hill AG. Systematic review of oxidative stress associated with pneumoperitoneum. Br J Surg. 2009;96(8):836-850.

6. Windberger UB, Auer R, Keplinger F, Langle F, Heinze $\mathrm{G}$, Schindl M, Losert UM. The role of intra-abdominal pressure on splanchnic and pulmonary hemodynamic and metabolic changes during carbon dioxide pneumoperitoneum. Gastrointest Endosc. 1999;49(1):84-91.

7. Malbrain ML, De laet I. It's all in the gut: introducing the concept of acute bowel injury and acute intestinal distress syndrome. Crit Care Med. 2009;37(1):365-366.

8. Giger UF, Michel JM, Opitz I, Th Inderbitzin D, Kocher T, Krahenbuhl L, Swiss Association of L, et al. Risk factors for perioperative complications in patients undergoing laparoscopic cholecystectomy: analysis of 22,953 consecutive cases from the Swiss Association of Laparoscopic and Thoracoscopic Surgery database. J Am Coll Surg. 2006;203(5):723-728.

9. Hasson HM, Galanopoulos C, Langerman A. Ischemic necrosis of small bowel following laparoscopic surgery. JSLS. 2004;8(2):159-163.

10. Wassenaar EB, Raymakers JT, Rakic S. Fatal intestinal ischemia after laparoscopic correction of incisional hernia. JSLS. 2007;11(3):389-393.

11. Uen YH, Liang AI, Lee HH. Randomized comparison of conventional carbon dioxide insufflation and abdominal wall lifting for laparoscopic cholecystectomy. J Laparoendosc Adv Surg Tech A. 2002;12(1):7-14. 